that inflammation of anasarcous legs has been as often subdued as provoked by acupuncture or incision ; that inflammation is much less likely to follow incisions in cases of renal than cardiac dropsy, when the circuIation is much impeded by valvular disease; and that an incision made with the spring scarifier is as safe as acupuncture, and much less painful.

The copious secretion of urine which usually follows a discharge of dropsical fluid through incisions in the skin admits of nearly the same explanation as that which I have already given you of the like phenomenon after the action of a hydragogue purgative. The escape of the dropsical effusion through one or more incisions in the skin, removes pressure. from the veins, and permits the blood to move more freely through the vessels. This greater freedom of the circulation is attended by a quickened absorption, and some of the absorbed dropsical liquid, more or less charged with urea, enters the circulation, and exerts a diuretic influence on the kidney. In the treatment of a copious dropsical effusion the main object, and the chief difficulty, is to overcome the vis inertia of the stagnant liquid. If once we can set the liquid in motion, whether by a primary diuretic action upon the kidneys, by first exciting a free discharge of liquid through the bowels, or by giving exit to a portion of the liquid through incisions in the skin-in which ever way the current is started-the outward movement of liquid often continues until the whole of the dropsical effusion has been swept away, and in each case a free secretion of urine constitutes a part of the eliminative process.

The anæmia of chronic Bright's disease is to be counteracted by a carefully regulated diet, and by the persevering use of one or other of the preparations of iron. When with anæmia there is a scanty secretion of urine, and a tendency to dropsy, a very useful combination is twenty minims of the tincture of perchloride of iron, with a drachm of spirit of nitrous rether, and a drachm of infusion of digitalis, or from ten to twenty minims of the tincture of digitalis in an ounce of water, given three times a day, soon after food. If the mixture be found to constipate, from half a drachm to a drachm of sulphate of magnesia may be added to each dose, or the bowels may be acted upon by an occasional dose of the compouud colocynth pill. Mercury in any form often acts powerfully and injuriously in cases of Bright's disease. The chief use of mercurials in these cases is to assist the operation of saline or vegetable purgatives.

Syphilitic symptoms, when present, are best treated by gradually increasing doses of iodide of potassium, with bark, or quinine. The cal. omel vapour bath has been found a useful remedy in some syphilitic cases.

When chronic renal disease is associated with a strumous diathesis, cod-liver oil may often be given with advantage.

Dyspncea is one of the most frequent and distressing symptoms asso. ciated with advanced Bright's disease. It has various causes, and requires various remedies. When it results from œdema of the lungs, or dropsical hydrothorax, it is best treated by the remedies for dropsy. In some cases, anæmia appears to be the chief cause of dyspncea. The red blood-corpuscles are the oxygen-carriers. When the blood-whether in cases of chlorosis or of Bright's disease-contains an excess of water with a corresponding deficiency of red corpuscles, the defective oxida tion of the tissues and the demand for air are manifested by hurried and laborious breathing. The remedy for this form of dyspnoa is to be sought for in the elimination of water, a carefully regulated nutritious diet, and iron as a restorative tonic.

When dyspncea results from pulmonary congestion and bronchial catarih, it is best treated by warm baths, fomentations, or poultices to the chest, and mild counter-irritants.

Paroxysmal dyspnœa, in many cases of advanced Bright's disease appears to result from the influence of deteriorated and poisoned blood upon the nerves and the nervous centres. The heart's action is rapid and feeble, the breathing is distressed and hurried, while the respiratory murmur is loud, puerile, and unattended by wheezing or crepitating sounds. This form of dyspnœa is usually more common and more distressing at night. It is not improbable that, in some cases, cardiac weakness, with palpitation and dyspnoea, may result from excessive contraction of the minute branches of the coronary arteries, excited by the stimulant action of morbid blood, and causing ancemia and malnutrition of the muscular walls of the heart. These symptoms are most effectually warded off by a carefully regulated diet, by promoting the action of the skin and bowels, and so far as possible of the kidneys, the object being to free the blood from accumulated impurities. Temporary relief is often afforded by ether, or by brandy. In some cases, a small dose of chloral hydrate, not more than ten grains, repeated at intervals of six or eight hours, is very beneficial. The chloral has this advantage over every preparation of opium, that it has no astringent action on the bowels, and that it rather increases than checks the secretion of the kidneys. It therefore never excites the distressing sickness which often results from the astringent influence of opiates in the advanced stages of renal degeneration. The muscular twitchings, and the painful cramps, which are common results of uræmia, and not unfrequent precursors of convulsions, may sometimes be kept off, or much mitigated, by twenty-grain doses of bromide of potassium, given twice or three times in the twenty-four hours. A combination of chloral hydrate with bromide of potassium appears sometimes to have a powerful influence in warding off uræmic convulsions.

Although the use of opium in all forms and stages of Bright's disease requires extreme care, on account of its tendency to check all the secretions except that of the skin, yet you will occasionally meet with cases in which the distressing nervous symptoms resulting from uræmia are relieved by opiates more effectually than by any other means. There are cases in which the carefully-observed result of a cautiously conducted experiment is sometimes a better guide than theory.

The sufferers from Bright's disease are always dyspeptics, and the gastric symptoms are often very obstinate and distressing. When, in consequence of renal degeneration, the blood is contaminated by retained urinary excreta, there is often a vicarious excretion of these impurities by the mucous membrane of the stomach and bowels. The gastric secretions are mingled with the ammoniacal products of decomposing urea ; digestion is consequently impaired; there are flatulent distension of the stomach and bowels, nausea, vomiting, and diarrhœa. Relief is to be sought by a carefully regulated diet, and by giving with the food from ten to twenty drops of dilute hydrochloric acid with a vegetable bitter. The liquor strychnix in doses of five minims, or the tincture of nux vomica in ten-minim doses, with a mineral acid, is sometimes especially efficacious. Pepsine may sometimes be given with ad. vantage.

In some cases of advanced renal degeneration, the vomiting is so incessant, that the patient has to be sustained by nutritive enemata, while iced water only, or iced milk in small quantity, is taken by the stomach. In some instances that have come under my observation, the straining and exhausting efforts of vomiting have been checked only by frequent whiffs of chloroform vapour.

When the stomach is very irritable, chloral can rarely be retained, it acts at once as an emetic ; but injected into the rectum, its soothing influence is very similar to that of chloroform vapour, while it has the advantage of producing a more durable impression on the nervous system, and therefore requires to be less frequently repeated.

When the retina is the seat of hæmorrhage, or of albuminuric retinitis, the eyes should be allowed to rest, and they should be carefully shaded from excess of light. Recovery of sight, more or less complete, may occur, but it is doubtful whether treatment has much influence upon the symptoms. My colleague Mr. Soelberg Wells, with whom I have seen several cases of this affection, strongly recommends moderate local bleeding by the artificial leech. When, on account of the anæmic condition of the patient, the abstraction of blood is undesirable, he has seen marked improvement follow the application of the dry cup to the temple, at intervals of five or six days. (A Treatise on the Diseases of the Eye. By J. Soelberg Wells. Second edition, p. 36r.)

\section{CASE OF STRANGULATED OBTURATOR HERNIA.}

\section{By CHARLES MAYO, F.R.C.S.,}

Consulting Surgeon to the Royal Hants County Hospital, Winchester.

JEMIMA H., aged 59, of rather spare habit and middling stature, acting as assistant matron at an institution for the reception of unfortunate or fallen women, called the Refuge, in this city, had been subject to occasional attacks, during the last two years, of pains across the bowels, with sickness and more or less constipation, from which she was relieved by mild aperients, injections, etc. On January 2oth, I was called to her on account of one of these attacks, late in the evening; and, as she had used an injection with some success, I gave her a mixture containing liquor ammoniæ acetatis, tincture of opium, and compound tincture of lavender, to be taken every three hours till the pain abated. Next day, the attack had passed off; but she spoke of considerable uneasiness which she felt in the left thigh. On February 27 th, the pain and sickness returned with renewed aggravation. Enemata with common salt, coarse sugar, and croton-oil, were given, with the hope of inducing downward action; and effervescing saline mixture with hydrocyanic acid and liquor opii sedativus, to abate the sickness. The treatment proved unavailing, and she sank exhausted on March 2nd.

On March $3 \mathrm{rd}$, my son, who assisted me in the above treament, took the greatest share in a post mortem investigation. The liver, spleen, and pancreas, were natural. We next examined the pyloric 
extremity of the stomach, as, from the pain and uneasiness which she had felt there, and the frequent vomiting, we suspected that some morbid condition would be discovered in that part. A ligature was placed on it, and another on the duodenum. The intervening portion was then removed and slit up, without showing more than ordinary vascularity or thickening. We then directed our attention to the small intestines, which were shrunken to about the size of the ring-finger, and quite empty. We then discovered, about two-thirds down, a loop three or four inches in length, when disentangled from the upper fourth of the left obturator foramen, where it was evidently strangulated, and required some force to withdraw it. The mid-portion of the loop was swollen and dark-coloured, and sufficiently distended with fæcal fluid to account for its not returning to its natural position, as it was sup. posed to have done in former attacks.

I am told that obturator hernia is not uncommon; but I have been able to find but few records of its occurrence. Sir William Lawrence, in his excellent treatise on Hernia, speaks of reports from two or three foreign authors, but has doubts of their authenticity as cases of obturator hernia. The clearest case of this malady that I can find is that reported by Mr. Hilton, Surgeon to Guy's Hospital, at p. 323 of vol. xxxi of the Medico.Chirurgical Transactions, where the symptoms were very analogous to those above described in our case ; and, as there were no external indications, their cause could not be revealed but by post mortem examination. As in Mr. Hilton's case, so in mine, there was room on the right side for the escape of a similar portion of intestine by the obturator foramen, which would allow the passage of the first joint of the forefinger easily ; and, indeed, the right side had been referred to as the seat of pain in some of her previous attacks. My nephew, Dr. Druitt, in his Vade Mecum or Manual of Modern Surgery', refers to a case by Mr. Obré, reported in Ranking's Abstract, vol. xiv, in which, a slight swelling being observed in the right thigh, Mr. Obré cut down upon it three inches below Poupart's ligament, and discovered a sac under the pectineus muscle, which he opened, and small intestine rose up. He then divided the stricture, and returned the gut through the obturator opening; and the case did well.

I have been anxious to forward this case to the BRITISH MEDICAL JOURNAL, as being one of its oldest subscribers ; and to say that, however frequent such cases may have been, yet I have never met with one during more than fifty years that $I$ have been in practice and attached to the County Hospital in this city. I shall be pleased to hear what any of my cotemporaries may be able to report on such cases. I do not remember to have seen such palpable openings above the obturator muscles, leaving room for a protrusion of peritoneum to form a sac for such hernia, as the vessels and nerves appeared to fill the notch in the bone, and the peritoneum passed smoothly over them.

Professor Miller, in a second edition of his Practice of Surgery (Edinburgh, 1852), gives a meagre account of this hernia; while Samuel Cooper, in his Dictionary (1809) and his First Lines (1820), gives excellent descriptions, and quctes from Sir Astley Cooper several varieties and the difficulties of discovering the seat of obstruction during life.

I might have mentioned that our patient was often employed in laundry work, or, at any rate, superintending; and that she attributed the painful attacks above mentioned to her reaching up to hang up clothes to dry on a line. This might have induced a downward action of the diaphragm, so as to more or less force down the bowels at certain intervals.

\section{CASE OF TRAUMATIC TETANUS TREATED WITH BROMIDE OF POTASSIUM : RECOVERY.}

By CHARLES ROBERT THOMPSON, Esq., Westerham.

ON January $24^{\text {th }} 1873$, J. H., aged 35 , a robust, healthy man, by trade a baker and grocer, was thrown from a cart, which capsized by collision with another, pitching on his right hand. He was slightly cut and bruised about the face.

I found complete dislocation of the hand backwards, without any fracture as far as I could determine by close examination, with a small jagged wound of the skin over the end of the ulna. I did not make out that this wound extended into the joint. The loss of blood was trifling ; and there was no loss of nervous sensibility.

After carefully cleansing the wound, the hand was brought into position by straight extension, the forearm being bent, and counterextension applied at the elbow; a straight splint was applied from the elbow to the ends of the fingers, at the back of the arm ; and oiled lint was placed on the wound.

He remained in bed a few days, being much shaken and bruised; the wrist was painful and swollen, with some very offensive discharge from the wound, on account of which it was poulticed. On the tenth day, I found the parts above the wrist inflamed and boggy, and made an incision which gave exit to sloughy cellular tissue. Excepting that his nights were bad from pain in the wrist, his general condition was favourable; he took food well, walked out when the weather allowed, and seemed convalescing.

February I $3^{\text {th }}$ (twentieth day from injury). I found him sttting up, looking ill, and complaining that he had no sleep for two nights on account of pain in his back, between the shoulders, and in the neck and jaws. He had shivered very much the night before; and had sweated profusely. He was quite unable to open his jaw. Whilst I was examining the injured wrist, he groaned with pain in his back, indicating a point just below and between the scapulæ. The skin was sweating. Pulse 86, very soft. The bowels had been regular throughout; the urine was said to be scanty and turbid. He could swallow liquids but slowly and with difficulty. The parts above the wrist were still inflamed and painful ; and a boggy place which did not seem relieved by the former incision was now freely opened, and the poultice continued. I ordered him to go to bed directly; to take a dose of castor-oil in brandy; to have his back well and frequently rubbed with a stimulating liniment; to be fed every two hours, alternately with beef-tea and milk or gruel containing brandy; and for medicine, bromide of potassium thirty grains, and spirit of chloroform twenty drops, in one ounce of water, to be taken every four hours.

February 14th. The paroxysms of pain in the back had been less severe. The nurse said that be seemed composed, and slept after each dose of the medicine, and had taken nourishment as ordered. The bowels acted freely with the oil. The tip of the tongue could be just made visible between the teeth, but not protruded. There was a free discharge of shreddy pus and cellular sloughs from the wounds.

February 2 Ist. The condition through the week had been good. There was steady diminution of pain in the kack, so that he got good sleep. He complained still of severe pain in the back, if anyone came into the room and talked to him; and more especially when the injured wrist was touched or dressed. He could now, with great care and deliberation, protrude about half an inch of tongue between his teeth; it was covered with thick creamy yellow fur. His breath smelt strongly of the peculiar odour of the bromide, something like a foul sponge ; this was perceptible through the room, showing how he was saturated with the salt. His strength was well maintained; he took nourishment well, increasing the brandy a little, or substituting port wine occasionally. The wounds on the arm were healthy; the sloughs were cleared out, so that water-dressing was applied instead of the poultice. He continued the medicine; and had the neck and face rubbed with belladonna liniment.

February $25^{\text {th. }}$ More pain in the hand had made him restless. A small abscess had formed on the dorsum of the hand, which I opened. There was slight improvement in the dorsal pains and in the locked jaw. He seemed more feeble. The dose of bromide of potassium was reduced to twenty grains every six hours, with two grains of quinine.

From this date, improvement went on steadily. The wounds about the wrist healed. The power of opening the jaw very slowly but very positively increased, so that by March 16 th he was able to protrude his tongue fully, though very slowly and with much effort. Pain would still come in the back if he sat up too long, or if anything excited him. At the end of March, he was quite convalescent, though weak, and was able to walk out daily, though with backache if he over-exerted him. self. He could now masticate solid food, but complained of pain in the muscles of the jaw if he tried them too long. The hand and wrist were quite sound, but stiff from want of use and from the mischief about the flexor tendons; they improved daily by passive motion and rubbing. The only medicine now given was quinine, two grains twice daily.

REMARKS.- Just before this case occurred, I had read in the JoURNAL (February 8th, p. I48) MI. Voisin's recommendation of bromide of potassium in tetanus, mentioning three cases of well marked traumatic tetanus in which cure was effected by the bromide in large doses and subcutaneous injection of morphia. I determined at once to use a drug which seems to commend itself so thoroughly by the extraordinary control which it possesses over the convulsions of epilepsy; and the result has been most satisfactory. Whether the case would have run on to acute tetanus, as it seemed to promise to do, but for the remedy, it is not possible to say; but the relief obtained immediately from the bromide was most decided and unmistakable. The whole amount given between February $13^{\text {th }}$ and March $25^{\text {th }}$ was six and a half ounces : the first ten days, three drachms daily; then two drachms till March 15th; after that, one drachm. The only effect it seemed to 